Original Paper http://ajol.info/index.php/ijbcs http://indexmedicus.afro.who.int

\title{
Connaissances traditionnelles des plantes locales utilisées contre les bio-agresseurs des cultures et produits agricoles chez le peuple Turka au Burkina Faso
}

\author{
Soumaïla SOURABIE ${ }^{\mathbf{*}}$, Patrice ZERBO ${ }^{\mathbf{1}}$, Djibril YONLI ${ }^{\mathbf{2}}$ et Joseph I. BOUSSIM ${ }^{\mathbf{1}}$ \\ ${ }^{1}$ Laboratoire de Biologie et Ecologie Végétales, Université Joseph KI-ZERBO, Ouagadougou, Burkina Faso. \\ ${ }^{2}$ Laboratoire de Malherbologie, Institut de l'Environnement et de Recherches Agricoles, Ouagadougou, \\ Burkina Faso. \\ *Auteur correspondant ; E-mail : soumismo@gmail.com
}

\section{RESUME}

La présente étude a pour objectif de faire l'état des connaissances traditionnelles endogènes relatives aux plantes locales utilisées contre les bio-agresseurs des cultures et des produits agricoles. A travers une série d'enquêtes ethnobotaniques réalisée de 2018 à 2019 dans la région des Cascades au Burkina Faso, 150 personnes appartenant à l'ethnie Turka ont été interviewées. Les informations recherchées en utilisant des entretiens semidirectifs, ont concerné les noms locaux des plantes, les parties utilisées, les domaines d'utilisation et leurs modes d'utilisation. Les données collectées ont permis de recenser 33 espèces de plantes réparties en 30 genres et 19 familles utilisées traditionnellement contre les bio-agresseurs agricoles. Ces plantes interviennent sélectivement dans trois domaines: la répulsion des insectes, l'inhibition des herbes envahissantes et la construction des greniers. Les espèces sont utilisées soit directement ou transformées en poudre ou en cendre. Les feuilles sont plus utilisées que les autres organes. Cassia nigricans est l'espèce la plus utilisée pour son effet répulsif et Parkia biglobosa pour son effet inhibiteur. Des études approfondies sur les propriétés chimiques des potentielles espèces permettront de réduire l'impact des produits chimiques en agriculture contre les bio-agresseurs.

() 2020 International Formulae Group. All rights reserved.

Mots clés : Ethnobotanique, diversité végétale, ennemi agricole, secteur sud-soudanien, Afrique de l'ouest

\section{Traditional knowledge of local plants used against pests of crops and agricultural products among the Turka poeple in Burkina Faso}

\begin{abstract}
The objective of this study is to provide an overview of endogenous traditional knowledge relating to local plants used against pests of crops and agricultural products. Through an ethnobotanical's surveys carried out from 2018 to 2019 in the Cascades area in Burkina, 150 Turka ethnic group people were interviewed. Through semi-structured interviews, information looking for were concerned plants local names, their parts used, the domains and the modes of use. Data collected allowed to identify 33 plants' species belonged to 30 genera and 19 families, used traditionally against agricultural pests. These plants are selectively involved in three domains: repelling insects, inhibiting invasive weeds and building lofts. Species are used either directly or processed into powder or ash. The leaves are used more than other organs. Cassia nigricans is the most used species for its
\end{abstract}


repellent effect and Parkia biglobosa for its inhibitory effect. In-depth studies on the chemical properties of potential species will reduce the impact of chemicals in agricultural against pests.

(C) 2020 International Formulae Group. All rights reserved.

Keywords: Ethnobotany, plant diversity, agricultural pest, South Sudanese sector, West Africa

\section{INTRODUCTION}

L'augmentation rapide des populations des pays en développement entraine de nombreux problèmes parmi lesquels, la sécurité alimentaire (Golay, 2010). Pour y pallier, la plupart de ces pays consacrent une bonne partie de leur budget à l'agriculture, car son développement constitue une approche de solution pour assurer la sécurité alimentaire aux populations (Dipama, 2014). Cependant, la production et la conservation des productions agricoles sont toujours confrontées aux nombreuses contraintes biotiques et abiotiques (Anjarwala et al., 2016). L'une des principales causes est la perte de rendement des cultures agricoles due à la pression des bio-agresseurs (Benmeddour et Fenni, 2018). Pour y faire face, les populations utilisent continuellement des pesticides chimiques dominés par les herbicides et les insecticides de synthèse. Selon la FAO et International Water Management Institute (2018) chaque année 4,6 millions de tonnes de pesticides chimiques sont pulvérisés dans l'environnement dans le monde et, pays en développement représentent $25 \%$ de l'utilisation mondiale des pesticides dans l'agriculture. Cette situation serait à l'origine de la perturbation de l'équilibre écologique de l'écosystème, de la dégradation et de la destruction de la santé, de la biodiversité et de l'environnement (Chérif et al., 2016). Malgré cette forte sollicitation, les producteurs font toujours face à des bio-agresseurs. Cela démontre les limites de ces méthodes modernes de lutte. Ainsi, ne serait-il pas sage de recourir aux pratiques endogènes des populations locales, capitalisées depuis plusieurs générations et respectueuses de l'environnement? En effet, les populations locales ont depuis toujours utilisé les plantes pour lutter contre les bio-agresseurs de cultures agricoles (Toumnou et al., 2012). L'emploi des extraits des plantes pour contrôler les ennemis des cultures, des denrées agricoles et de cultures maraichères, a été rapporté par plusieurs travaux en Afrique de l'Ouest (Yarou et al., 2017) et au Burkina Faso (Traoré et al., 2015 ; Savadogo et al., 2016).

Cependant, peu d'études ont abordé les questions de la gestion traditionnelle par les plantes des ennemis des cultures agricoles et de leurs produits récoltés dans le secteur phytogéographique Sud-soudanien du Burkina Faso. La plupart des travaux de cette zone abordant les connaissances ethnobotaniques des populations locales ont porté sur les plantes des galeries forestières (Bélem et al., 2010 ; Olivier et al., 2012), les plantes médicinales (Zerbo et al., 2013), les espèces oléagineuses (Tiétiambou et al., 2016). Pourtant, il est ressorti d'une enquête préliminaire dans la zone, que certains producteurs notamment de l'ethnie Turka, persistent de nos jours dans l'usage des plantes pour protéger leurs cultures et denrées alimentaires contre les bioagresseurs. L'objectif de la présente étude est de contribuer à une meilleure connaissance des ressources végétales exploitées par les producteurs Turka en vue de réduire l'effet de produits chimiques utilisés en agriculture. De façon spécifique, l'étude vise à inventorier les différentes espèces végétales et leurs modes d'utilisation, à évaluer l'importance d'utilisation des différents organes de plantes et à identifier les espèces les plus exploitées dans le milieu d'étude par les populations. 


\section{MATERIEL ET METHODES}

\section{Milieu d'étude}

La zone d'étude couvre les provinces de la Comoé $10^{\circ} 36^{\prime}$ Nord, $4^{\circ} 45^{\prime}$ Ouest et de la Léraba $10^{\circ} 40^{\prime} 00^{\prime \prime}$ Nord, $5^{\circ} 12^{\prime} 00^{\prime \prime}$ Ouest (Figure 1). Cette zone appartient à la région des Cascades qui s'étend sur une superficie de 18 $407 \mathrm{~km}^{2}$ (INSD, 2017). Elle appartient au secteur phytogéographique Sud-soudanien du Burkina Faso. La végétation y est constituée de savane boisée et arborée, les forêts claires entrecoupées de galeries forestières. Cette région bénéficie d'une pluviométrie supérieure à $1200 \mathrm{~mm}$ et compte parmi les zones les mieux arrosées du pays (Thiombiano et Kampmann, 2010). Le climat est caractérisé par deux saisons. La saison pluvieuse est courte et va de mai à octobre. Les sols généralement gravillonnaires, hydromorphes et ferralitiques offrent des conditions favorables aux activités agricoles dominées par les cultures pluviales dont les systèmes sont extensifs (INSD, 2017).

\section{Collecte des données}

Des enquêtes ethnobotaniques ont été conduites de novembre 2018 à juillet 2019 dans douze villages dont sept (7) de la Comoé (Bérégadougou, Bodadiougou, Kolokolo, Mondon, Moussodougou, Tarfila, Toumousseni) et cinq (5) de la Léraba (Douna, Malon, Niofila, Tourny, Wolokonto). Ces villages sont majoritairement peuplés de personnes d'ethnie Turka. Le choix de cette communauté se justifie par leur connaissance sur les espèces de plantes utilisées en agriculture et leur persistance dans cette pratique traditionnelle pour l'amélioration des productions agricoles et la conservation des denrées alimentaires.

Au total, 150 personnes, âgées de 45 à 85 ans, ont été interviewées individuellement. Ces enquêtées ont été choisies sur la base de leur adhésion volontaire et leur expérience dans l'utilisation des plantes locales dans le domaine agricole. La technique d'enquête utilisée était basée sur des interviews directes, utilisant un questionnaire semi-structuré préalablement testé (Alexiades, 1996). Les principales informations collectées au cours des entretiens ont concerné les plantes utilisées dans la gestion durable des cultures et produits agricoles, leurs noms locaux en langue locale Turka, les domaines d'usage des espèces végétales, les organes utilisés et leurs modes d'utilisation. Des échantillons de ces espèces végétales ont été concomitamment prélevés dans le milieu avec l'aide de personnes ressources herboristes de la localité.

\section{Identification des espèces}

Les espèces inventoriées ont été directement identifiées sur le terrain à l'aide de la Flore du Sénégal (Berhaut, 1979) et d'autres ouvrages pseudo-scientifiques tels que Arbres, arbustes et lianes de la zone soudanosahélienne (Arbonnier, 2009). Les spécimens de celles qui ont fait l'objet d'un doute, ont été déterminés grâce à ceux de l'Herbier Université de Ouagadougou (Herbier OUA). La nomenclature adoptée est celle du catalogue des plantes vasculaires du Burkina Faso (Thiombiano et al., 2012). Les types biologiques ont été également déterminés.

\section{Analyse des données}

Les données collectées ont été manuellement dépouillées puis saisies et traitées dans le logiciel Sphinx Plus. Le tableur Excel a été ensuite utilisé pour la réalisation des tableaux et l'expression graphique (diagramme, histogrammes).

Pour apprécier la valeur d'usage des espèces recensées et le niveau de connaissance des populations, les indices suivants ont été calculés :

- $\quad$ le Degré de Fidélité (DF) : il a été calculé pour chaque espèce et organe utilisé par les populations pour lutter contre les bioagresseurs, suivant la formule : $\mathrm{DF}=\frac{F c}{N} \times 100$, avec $\mathrm{Fc}$ désignant le 
nombre d'enquêté qui ont cité l'espèce et $\mathrm{N}$ le nombre total de personnes enquêtées (Teklehaymanot et al., 2007).

- la valeur d'usage ethnobotanique (VUE) : la valeur d'usage de chaque plante identifiée (Use Value species ou $U V s$ ) a été calculée selon la formule simplifiée de Cotton (1996): VUE $=\frac{\Sigma \mathrm{Ui}}{\mathrm{Ut}}$ où, $U i$ est le nombre de citation par espèce et, $U t$ représente le nombre d'informateurs.

- le Facteur de Consensus Informateur $(F C I)$ : le niveau de connaissance des populations sur les usages des espèces végétales dans les systèmes agricoles a été apprécié sur la base du calcul du Facteur de Consensus Informateur $(F C I)$ ou Informant Consensus Factor défini par Andrade-Cetto et Heinrich (2011). $F C I=\frac{N u r-N t}{N u r-1}$ où, Nur correspond au nombre de citation pour chaque catégorie et $N t$ représente le nombre d'espèces pour cette même catégorie. Les valeurs du FCI sont comprises entre 0 et 1 . Une valeur élevée de FCI proche de 1, indique qu'une grande proportion d'informateurs mentionnent un nombre réduit d'espèces végétales dans une catégorie spécifique. À l'inverse, une valeur faible (proche de 0 ) indique une grande diversité d'espèces dans une catégorie d'usage citée par les informateurs

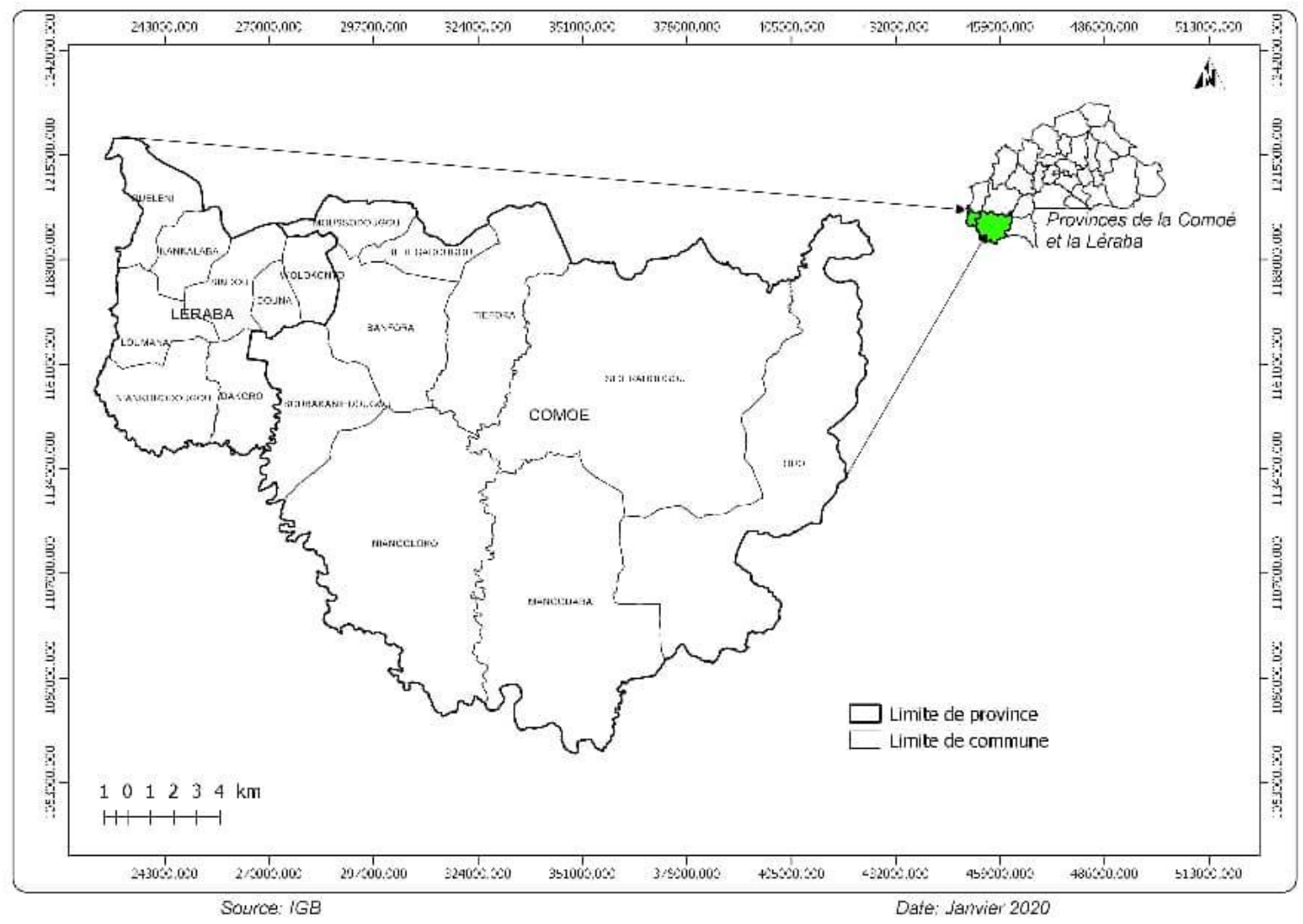

Figure 1 : Carte de la zone d'étude : provinces de la Comoé et la Léraba. 


\section{RESUSLTATS \\ Diversité floristique}

Les informations collectées ont permis d'identifier 33 espèces végétales utilisées dans les processus agricoles par les populations Turka au Burkina Faso. Cette flore constituée de ligneux et d'herbacées, appartient à 19 familles et 33 genres (Tableau 1). La famille des Poaceae est la plus représentée avec cinq (5) espèces, suivie par les Fabaceae-Faboideae et des Rutaceae avec respectivement quatre (4) et deux (2) espèces. Ces espèces recensées appartiennent à cinq (5) types biologiques: Géophytes, Phanérophytes, Chaméphytes, Hémicryptophytes et Thérophytes (Figure 2). Les Phanérophytes (61\%) suivi des Thérophytes (24\%) sont plus sollicités.

\section{Domaines d'usage et indices ethnobotaniques des espèces}

Les savoirs endogènes sur l'usage des plantes locales utilisées dans la lutte contre les bio-agresseurs en agriculture sont répartis en trois (3) domaines : la lutte contre les insectes des cultures en champs et des denrées alimentaires stockés après récolte, la lutte contre les herbacées envahissantes et la construction des greniers traditionnels (Tableau 2). En effet, l'examen des résultats révèle que $46 \%$ des espèces protègent contre les insectes tandis que 38\% entrent dans la construction des greniers traditionnels. Le calcul du facteur de consensus d'information $(I C F>0,5)$ montre que les populations s'accordent autour des usages des espèces végétales dans la conservation des produits agricoles stockés et la construction des greniers traditionnels.

Chez les Turka, six (6) espèces appartenant chacune à une famille et un genre (Tableau 1), sont utilisées pour protéger les cultures contre les herbacées envahissantes. Il s'agit de Tephrosia purpurea, Euphorbia hirta, Neocarya macrophylla, Azadirachta indica, Parkia biglobosa et Eucalyptus camaldulensis. Parkia biglobosa $(0,44)$ et Tephrosia purpurea $(0,22)$ sont les espèces les plus utilisées aux vues de leur valeur d'usage ethnobotanique (VUE). Pour repousser les insectes qui s'attaquent aux cultures et aux produits agricoles en stock dans les greniers, les populations ont recours à 17 espèces végétales réparties en 17 genres et 12 familles. Cassia nigricans $(0,52)$ et Zanthoxylum zanthoxyloides $(0,30)$ sont les plus utilisées parmi ces espèces. Dans la construction des différentes parties des greniers traditionnels, les populations utilisent sélectivement 14 espèces végétales réparties en 10 familles et 14 genres. Ainsi, les Turka utilisent plus Andropogon gayanus $(0,17)$ pour la confection de la toiture, Piliostigma thonningii $(0,17)$ pour la charpente et Neocarya macrophylla $(0,11)$ et Vitellaria paradoxa $(0,17)$ en mélange avec le banco du grenier.

\section{Organes utilisés et leurs modes d'utilisation}

Divers organes de plantes (Tableau 1) sont utilisés dans la lutte contre les bioagresseurs des cultures ou de leurs produits en stockage; il s'agit des feuilles, des tigesfeuillées, des écorces du tronc, des fruits, des bulbes, des rhizomes et même des plantes entières. Le calcul de leurs degrés de fidélité (Figure 3) révèle que les feuilles (42\%) sont majoritairement sollicitées, suivies des écorces du tronc $(21 \%)$. Les bulbes et les rhizomes ne représentent chacun que $4 \%$ des espèces inventoriées. Ces organes peuvent être utilisés directement ou transformés en poudres principalement ou extraits aqueux. Les principales formes d'utilisation rencontrées sont les poudres $(53 \%)$ et les extraits aqueux (47\%). Le séchage et la calcination sont les différends procédés d'obtention de la poudre. Seuls ou associés, ces organes de plantes interviennent dans la préparation de plusieurs recettes pour réduire la pression des bioagresseurs.

$\mathrm{Au}$ niveau des plantes à effets inhibiteurs sur les herbes envahissantes (Tableau 1), les populations utilisent plus les feuilles (37\%) et les écorces du tronc (19\%). Et pour repousser les insectes qui s'attaquent aux produits agricoles en stock dans les greniers, les populations font recours à la tige feuillée (Photo 1), à l'écorce du tronc (Photo 2) et aux bulbes des plantes.

Pour la construction des greniers, les organes employés sont le tronc (26\%), les tiges feuillées $(21 \%)$, les branches et les fruits $(16 \%$ chacune) et les feuilles (11\%). 
S. SOURABIE et al. / Int. J. Biol. Chem. Sci. 14(4): 1390-1404, 2020

Tableau 1 : Liste des espèces végétales inventoriées, types biologiques, domaines d'usage et valeurs d'utilisation.

\begin{tabular}{|c|c|c|c|c|}
\hline $\begin{array}{l}\text { Nom local en } \\
\text { Turka }\end{array}$ & $\begin{array}{l}\text { Genres et espèces } \\
\text { Familles }\end{array}$ & $\begin{array}{l}\text { Types } \\
\text { biologiques }\end{array}$ & Domaines d'usage & VUE \\
\hline Laïw & $\begin{array}{l}\text { Allium sativum } \mathrm{L} . \\
\text { Liliaceae }\end{array}$ & Gé & Insectifuge par usage de la poudre & 0,03 \\
\hline Isiri & $\begin{array}{l}\text { Andropogon gayanus Kunth. } \\
\text { Poaceae }\end{array}$ & Hé & Construction du grenier traditionnel & 0,17 \\
\hline \multirow{2}{*}{ Sumayantuugou } & \multirow{2}{*}{$\begin{array}{l}\text { Azadirachta indica A. Juss. } \\
\text { Meliaceae }\end{array}$} & \multirow{2}{*}{$\mathrm{Ph}$} & Insecticide par usage des fruits et des feuilles & 0,05 \\
\hline & & & Herbicide par usage d'extraits d'écorce du tronc & 0,06 \\
\hline \multirow[b]{2}{*}{ Fissibitougu } & \multirow{2}{*}{$\begin{array}{l}\text { Bobgunnia madagascariensis } \\
\text { (Desv.) J.H.Kirkbr.\& Wiersema } \\
\text { Fabaceae-Faboideae }\end{array}$} & \multirow[b]{2}{*}{$\mathrm{Ph}$} & Insectifuge par usage des fruits & 0,05 \\
\hline & & & Construction des grenier traditionnels & 0,05 \\
\hline Sensenw & $\begin{array}{l}\text { Capsicum frutescens L. } \\
\text { Solanaceae }\end{array}$ & $\mathrm{Ch}$ & Insectifuge par usage de la poudre & 0,02 \\
\hline Menganjitougu & $\begin{array}{l}\text { Carica papaya } \mathrm{L} . \\
\text { Caricaceae }\end{array}$ & $\mathrm{Ph}$ & Insectifuge par usage des feuilles & 0,02 \\
\hline Tchobatiangou & $\begin{array}{l}\text { Cassia nigricans Vahl. } \\
\text { Fabaceae-Faboideae }\end{array}$ & $\mathrm{Th}$ & Insectifuge par usage de la poudre & 0,59 \\
\hline Vahacohlie & $\begin{array}{l}\text { Cissus quadrangularis L. } \\
\text { Vitaceae }\end{array}$ & $\mathrm{Ph}$ & Insectifuge par usage de la poudre & 0,02 \\
\hline Loumbroutougou & $\begin{array}{l}\text { Citrus limon } \mathrm{L} . \\
\text { Rutaceae }\end{array}$ & $\mathrm{Ph}$ & Insectifuge par usage de la poudre des feuilles & 0,01 \\
\hline Café hyengou & $\begin{array}{l}\text { Cymbopogon schoenanthus (L.) } \\
\text { Spreng. } \\
\text { Poaceae }\end{array}$ & Hé & Insectifuge par usage de la poudre & 0,05 \\
\hline
\end{tabular}


S. SOURABIE et al. / Int. J. Biol. Chem. Sci. 14(4): 1390-1404, 2020

\begin{tabular}{|c|c|c|c|c|}
\hline Fomw & $\begin{array}{l}\text { Digitaria exilis (Kippist) Stapf } \\
\text { Poaceae }\end{array}$ & Th & Construction du grenier traditionnel & 0,10 \\
\hline Kanli & $\begin{array}{l}\text { Elaeis guineensis Jacq. } \\
\text { Arecaceae }\end{array}$ & $\mathrm{Ph}$ & Construction du grenier traditionnel & 0,02 \\
\hline Filawotuugou & $\begin{array}{l}\text { Eucalyptus camaldulensis Dehnh } \\
\text { Myrtaceae }\end{array}$ & $\mathrm{Ph}$ & Herbicide par usage d'extrait des feuilles & 0,05 \\
\hline Nonmoutingou & $\begin{array}{l}\text { Euphorbia hirta L. } \\
\text { Euphorbiaceae }\end{array}$ & $\mathrm{Th}$ & Herbicide par usage d'extraits de la plante entière & 0,02 \\
\hline Tchogopougou & $\begin{array}{l}\text { Guiera senegalensis J.F.Gmel. } \\
\text { Combretaceae }\end{array}$ & $\mathrm{Ph}$ & Construction du grenier traditionnel & 0,03 \\
\hline Vahtiaingou & $\begin{array}{l}\text { Hyptis suaveolens (L.) Poit. } \\
\text { Lamiaceae }\end{array}$ & $\mathrm{Th}$ & Insectifuge par usage de la poudre des feuilles & 0,02 \\
\hline Koulougou & $\begin{array}{l}\text { Imperata cylindrica (L.) Raeusch. } \\
\text { Poaceae }\end{array}$ & Gé & Construction du grenier traditionnel & 0,05 \\
\hline Djanhanoutougou & $\begin{array}{l}\text { Jatropha gossypiifolia L. } \\
\text { Euphorbiaceae }\end{array}$ & $\mathrm{Ph}$ & Insectifuge par usage de la poudre des fruits & 0,01 \\
\hline Pianri & $\begin{array}{l}\text { Khaya senengalensis (Desr.) A. } \\
\text { Juss. } \\
\text { Meliaceae }\end{array}$ & $\mathrm{Ph}$ & $\begin{array}{l}\text { Insectifuge par usage de la poudre des écorces du } \\
\text { tronc }\end{array}$ & 0,03 \\
\hline Bin gnantingou & $\begin{array}{l}\text { Lantana camara } \mathrm{L} . \\
\text { Verbenaceae }\end{array}$ & $\mathrm{Ph}$ & Insectifuge par usage de la poudre & 0,02 \\
\hline Benbentougou & $\begin{array}{l}\text { Monotes kerstingii Gilg } \\
\text { Dipterocarpaceae }\end{array}$ & $\mathrm{Ph}$ & Construction du grenier traditionnel & 0,10 \\
\hline \multirow{2}{*}{ Sabiri } & Neocarya macrophylla (DC.) & \multirow[b]{2}{*}{$\mathrm{Ph}$} & Construction du grenier traditionnel & 0,11 \\
\hline & $\begin{array}{l}\text { Prance ex F.White } \\
\text { Chrysobalanaceae }\end{array}$ & & Herbicide par usage d'extrait des feuilles & 0,07 \\
\hline
\end{tabular}


S. SOURABIE et al. / Int. J. Biol. Chem. Sci. 14(4): 1390-1404, 2020

\begin{tabular}{|c|c|c|c|c|}
\hline Koumpiètougou & $\begin{array}{l}\text { Nicotiana tabacum L. } \\
\text { Solanaceae }\end{array}$ & $\mathrm{Th}$ & Insectifuge par usage de la poudre des feuilles & 0,05 \\
\hline Saplogou & $\begin{array}{l}\text { Parinari curatellifolia Planch. Ex } \\
\text { Benth. } \\
\text { Chrysobalanaceae }\end{array}$ & $\mathrm{Ph}$ & Construction du grenier traditionnel & 0,07 \\
\hline Lenhangou & $\begin{array}{l}\text { Parkia biglobosa (Jacq.) R.Br.ex } \\
\text { G.Don. } \\
\text { Fabaceae-Mimosoideae }\end{array}$ & $\mathrm{Ph}$ & Herbicide par usage des cosses du fruit & 0,44 \\
\hline Jaaga & $\begin{array}{l}\text { Pennisetum americanum (L.) Leeke } \\
\text { Poaceae }\end{array}$ & $\mathrm{Th}$ & Construction du grenier traditionnel & 0,05 \\
\hline kinkiengou & $\begin{array}{l}\text { Pericopsis laxiflora (Benth.) } \\
\text { Meeuwen } \\
\text { Fabaceae-Faboideae }\end{array}$ & $\mathrm{Ph}$ & Construction du grenier traditionnel & 0,05 \\
\hline Baagou & $\begin{array}{l}\text { Piliostigma thonningii (Schumach.) } \\
\text { Milne-Redh } \\
\text { Fabaceae-Caesalpinioideae }\end{array}$ & $\mathrm{Ph}$ & Construction du grenier traditionnel & 0,17 \\
\hline Tilougou & $\begin{array}{l}\text { Pteleopsis suberosa Engl. \& Diels } \\
\text { Combretaceae }\end{array}$ & $\mathrm{Ph}$ & Construction du grenier traditionnel & 0,07 \\
\hline \multirow{2}{*}{ N'gunow } & \multirow{2}{*}{$\begin{array}{l}\text { Tephrosia purpurea (L.) Pers. } \\
\text { Fabaceae-Faboideae }\end{array}$} & \multirow{2}{*}{$\mathrm{Th}$} & Herbicide par usage d'extraits de feuilles & 0,22 \\
\hline & & & Insectifuge par usage de la poudre & 0,05 \\
\hline Mousongou & $\begin{array}{l}\text { Vitellaria paradoxa C.F. Gaertn } \\
\text { Sapotaceae }\end{array}$ & $\mathrm{Ph}$ & Construction du grenier traditionnel & 0,17 \\
\hline Djatéri & $\begin{array}{l}\text { Zanthoxylum zanthoxyloides (Lam.) } \\
\text { Zepern. \& Timler Rutaceae }\end{array}$ & $\mathrm{Ph}$ & $\begin{array}{l}\text { Insecticide par usage de la poudre des écorces ou } \\
\text { des feuilles }\end{array}$ & 0,03 \\
\hline Gnamakou & $\begin{array}{l}\text { Zingiber officinale Roscoe } \\
\text { Zingiberaceae }\end{array}$ & $\mathrm{Th}$ & Insectifuge par usage de la poudre du rhizome & 0,02 \\
\hline
\end{tabular}

Légende : Gé= Géophyte ; Ph= Phanérophyte ; Th= Thérophyte ; Hé= Hémicryptophyte ; Ch= Chamephyte ; VUE= Valeur d'Usage des espèces 


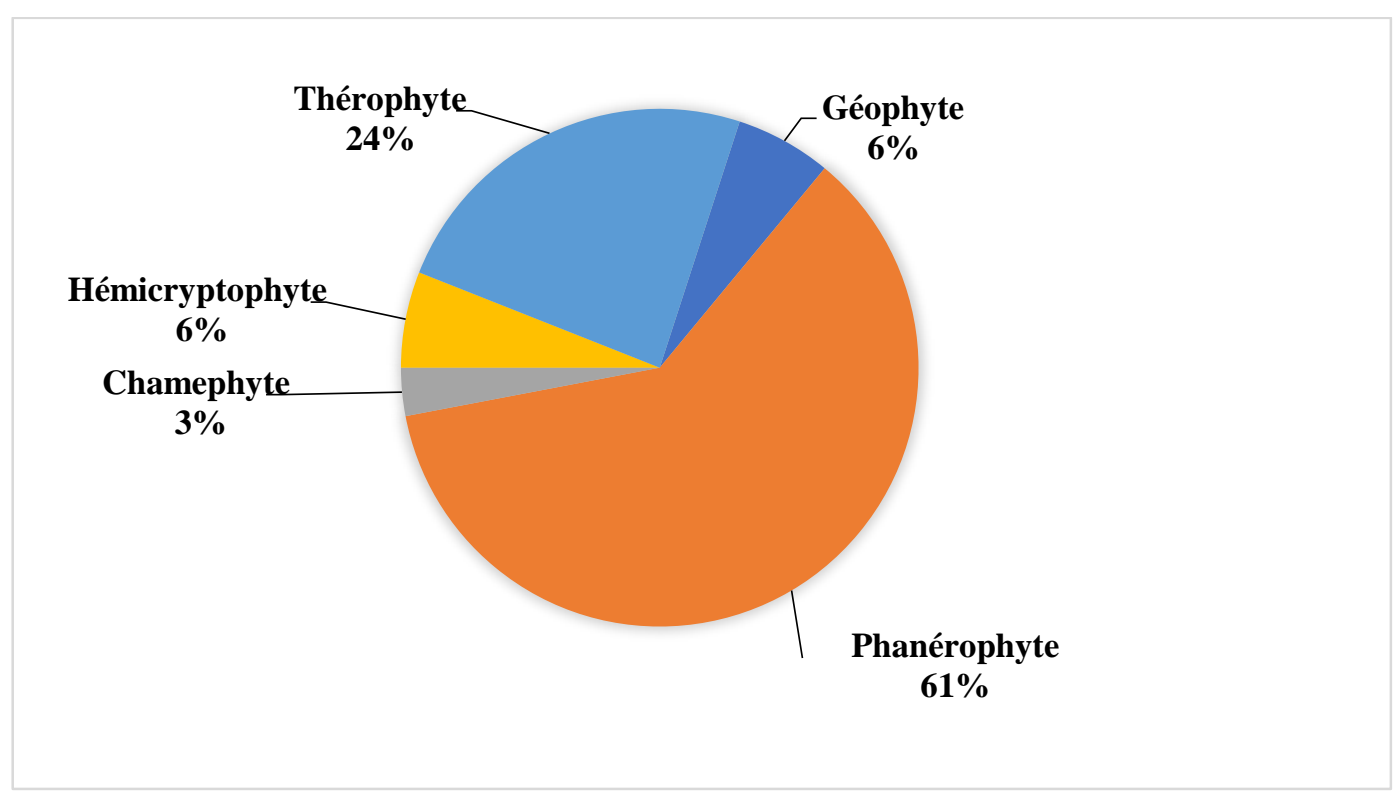

Figure 2 : Répartition des types biologiques.

Tableau 2 : Degrés de fidélité et facteurs de consensus d'information par domaines d'usage.

\begin{tabular}{lcc}
\hline Catégories d'usage & Degré de fidélité $\mathbf{( \% )}$ & FCI \\
\hline Lutte contre les herbes envahissantes & 16 & 0,44 \\
\hline Lutte contre les insectes & 46 & 0,75 \\
\hline Construction des greniers & 38 & 0,56 \\
\hline
\end{tabular}

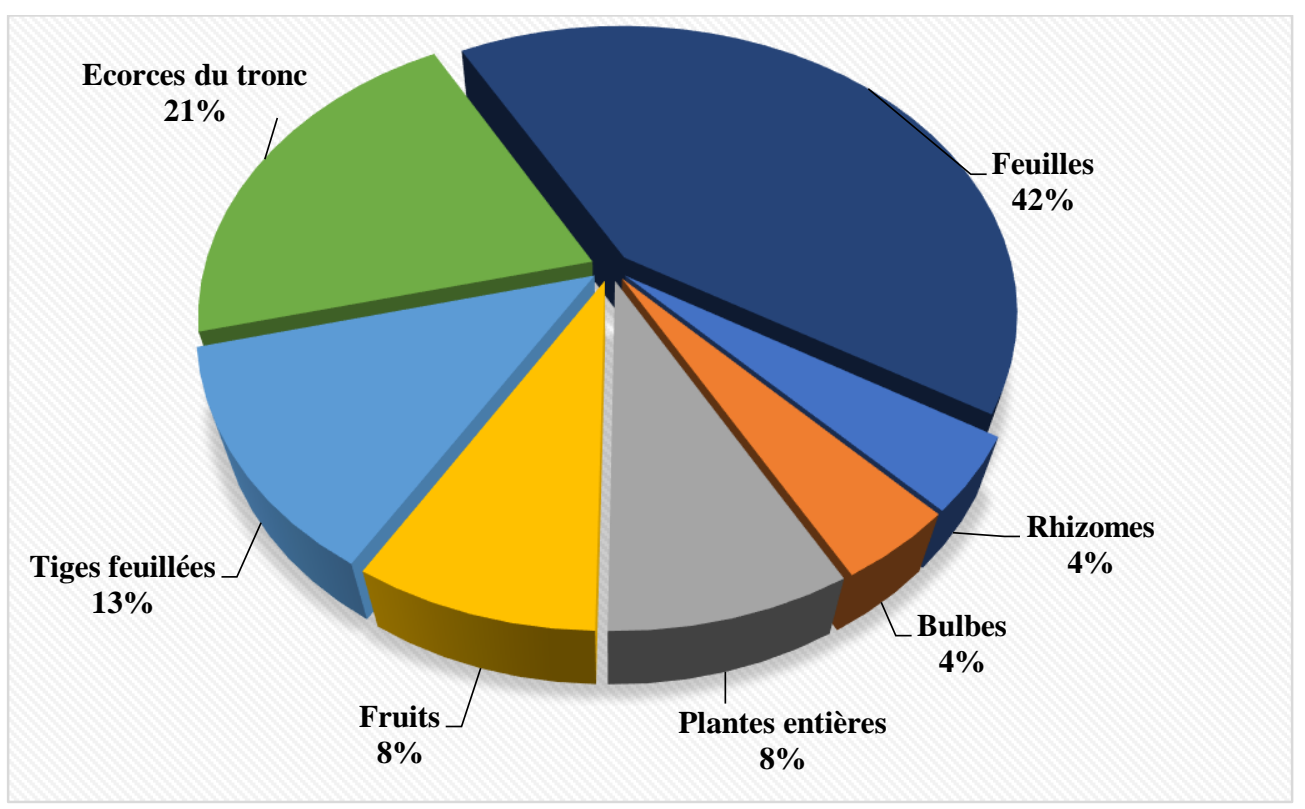

Figure 3 : Organes utilisés et leurs fréquences relatives de citation. 
S. SOURABIE et al. / Int. J. Biol. Chem. Sci. 14(4): 1390-1404, 2020

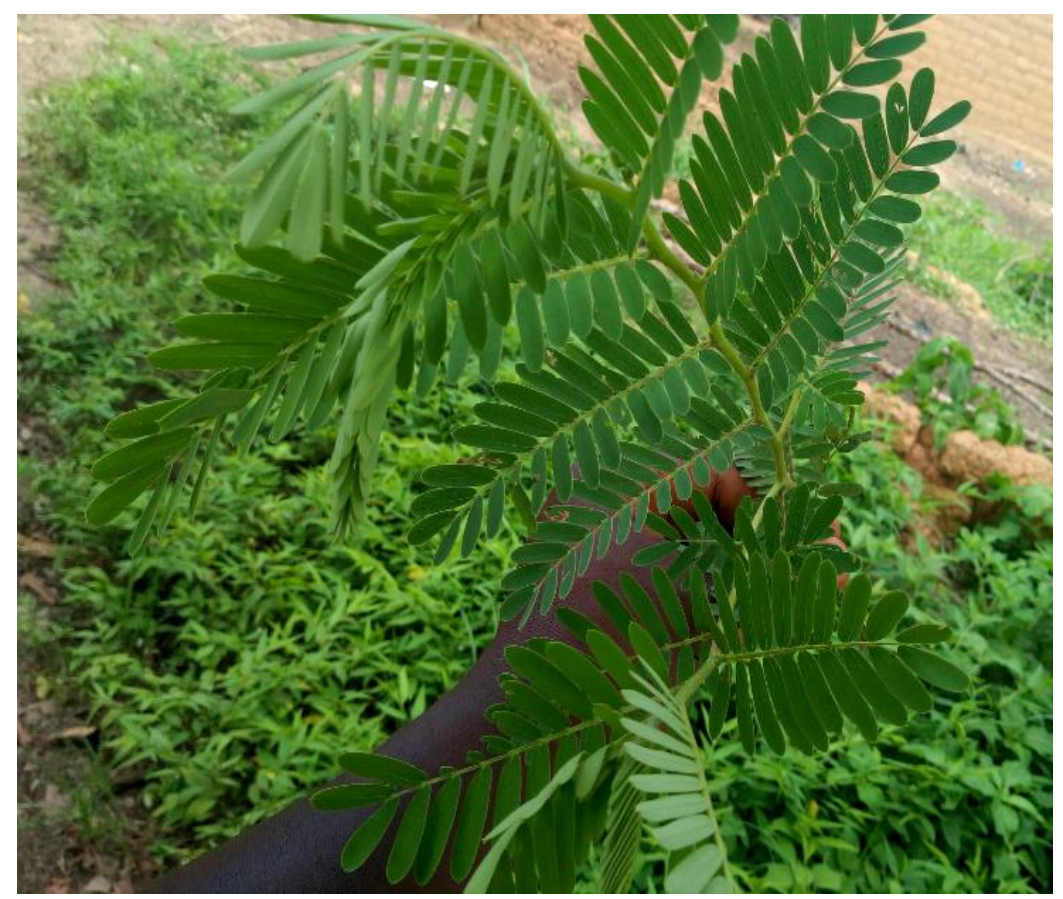

Photo 1 : Tige feuillée de Cassia nigricans.

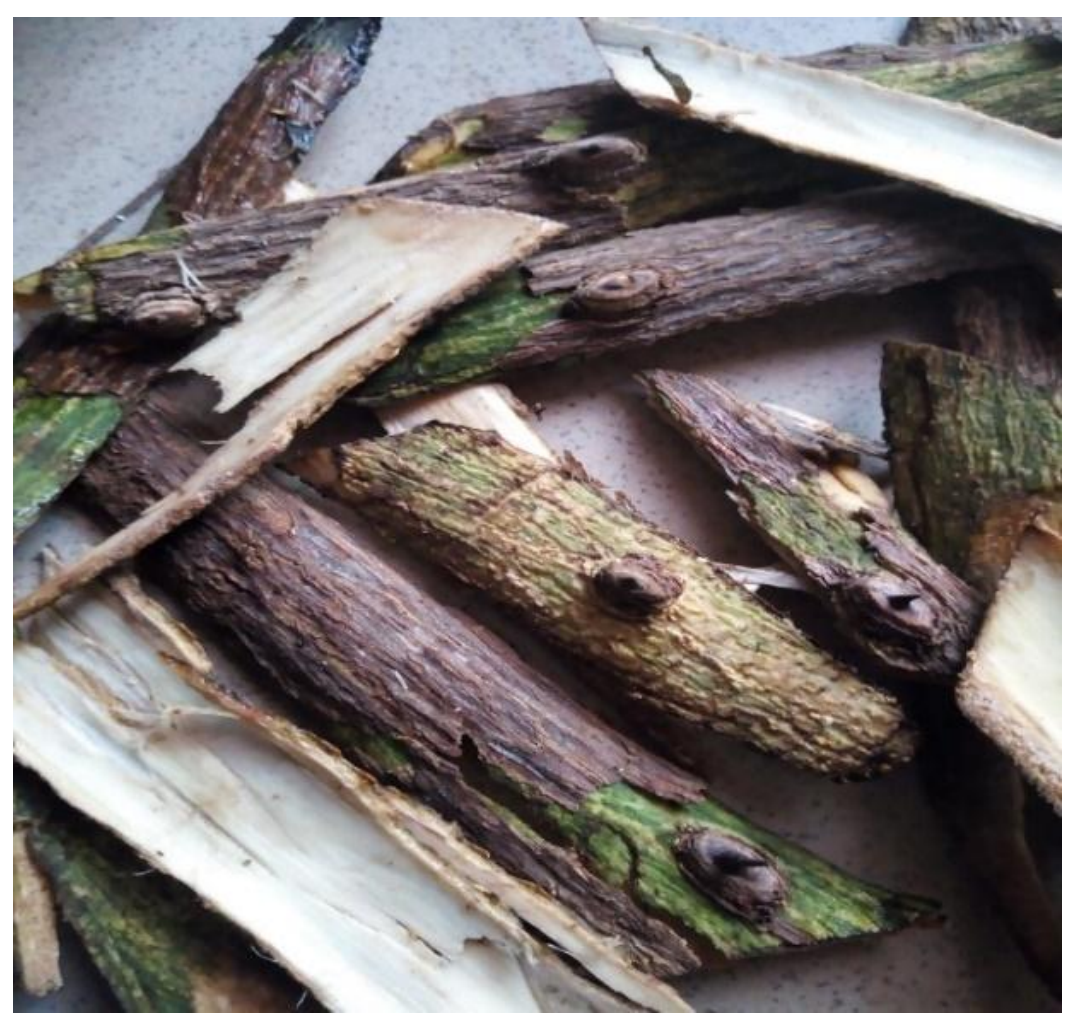

Photo 2 : Ecorce du tronc de Zanthoxylum zanthoxyloides. 


\section{DISCUSSION}

Trente-trois (33) espèces de plantes locales appartenant à 19 familles et 33 genres sont utilisées par les Turka dans la protection de leurs semences agricoles, des cultures au champ et les denrées stockées en grenier contre les insectes ravageurs. La localisation de la zone d'étude dans le secteur phytogéographique sud-soudanien justifierait cette richesse floristique. En effet, Savadogo et al. (2016) ont recensé 24 espèces de plantes traditionnellement utilisées par les Mossé pour lutter contre les insectes et les tiques dans le secteur subsahélien du Burkina Faso. Le niveau de connaissance qu'ont les Turka de l'usage des ressources végétales dans les systèmes agricoles pourrait également expliquer nos résultats. Ces résultats corroborent ceux de Toumnou et al. (2012) au Sénégal qui ont inventorié 35 espèces employées par les paysans pour repousser les insectes ravageurs des plantes céréalières et des légumineuses. En effet, la production agricole au Burkina Faso est dominée par les céréales notamment le sorgho, le mil, le maïs, le fonio et le riz (Herrera et Ilboudo, 2012). Cette forte diversité offre ainsi plusieurs possibilités dans le choix des espèces entrant dans la lutte contre les bioagresseurs.

Une analyse des résultats montre que les phanérophytes $(61 \%)$ sont beaucoup sollicités dans les luttes contre les bio-agresseurs du domaine agricole. Cette prédominance des phanérophytes dans la zone corrobore les résultats de Bélem et al., (2010), Traoré et al. (2011) et Olivier et al. (2012) relatifs aux plantes utilisées par les populations du Sudouest burkinabè. La disponibilité de ce type biologique dans le milieu aurait influencé donc les divers usages.

Chez les Turka du Burkina Faso, les espèces végétales impactent réellement le domaine agricole de par leurs actions inhibitrices des mauvaises herbes et répulsives des insectes. Les plantes à effets répulsifs semblent être bien connues des populations. En effet, la forte valeur de 1 'IFC $(I F C>0,5)$ témoignerait soit d'une efficacité de ces espèces soit d'une bonne connaissance des espèces par ce peuple. Au niveau de l'action inhibitrice, un faible consensus $(I F C<0,5)$ s'est dégagé autour de l'usage des espèces employées. La complexité du domaine de la lutte contre les herbacées envahissantes justifierait ce désaccord. Cherif et al. (2016) expliquent cet état par le développement des formes de résistance au traitement des bioherbicides des graines des adventices ou les plantules de certaines herbacées.

Une analyse des indices ethnobotaniques révèle un usage préférentiel des plantes par ce peuple Turka. En effet, certaines espèces (Cassia nigricans, Parkia biglobosa) ont des valeurs élevées tandis que d'autres présentent des valeurs faibles (Neocarya macrophylla). Cette variabilité du degré de satisfaction des populations vis-à-vis de ces ressources végétales serait due à la pression anthropique exercée sur ces espèces (Tiétiambou et al., 2016). De telles espèces méritent un recours aux mécanismes de gestion adéquat et durable afin de prolonger leur utilisation (Rives, 2012). Parkia biglobosa est l'espèce la plus utilisée (44\%) dans ce domaine. Ses feuilles, ses écorces du tronc et ses cosses ont de nombreuses formes d'utilisation dans le milieu Turka soit directement sans transformation préalable ou par leur réduction en poudre. Cette espèce tire son hégémonie de ses divers usages dans la satisfaction des besoins des populations. En effet, ses vertus médicinaux, alimentaires et insectifuges ont été rapportés par plusieurs travaux (Bélem et al., 2010; Traoré et al., 2011 ; Savadogo et al., 2016). Sa disponibilité dans la zone d'étude (Thiombiano et Kampmann, 2010) et sa facilité d'accès seraient à l'origine de sa sollicitation. Le pouvoir inhibiteur de la poudre de ses cosses a été également évoqué par les travaux de Kambou et al. (2000). Toutes ces actions témoignent de l'usage des plantes locales en agriculture (Sahu et Devkota, 2013). Cependant peu de travaux évoquent l'usage de Cassia nigricans dans les stratégies de lutte contre les ravageurs des denrées stockées.

Les degrés d'utilisation des organes sont également variables. Cependant une prédominance des parties périphériques des plantes (feuilles et écorces du tronc) est 
observée chez les populations. Pour certains auteurs (Zerbo et al., 2011), l'accès facile de ces organes serait à la base de cette forte sollicitation. D'autres auteurs par contre l'attribueraient à la présence de métabolites secondaires, matériaux de base contenus dans ces parties (Maloueki et al., 2015). Quant aux modes de leur prélèvement, la forme pratiquée chez Cassia nigricans, basée principalement sur l'arrachage de la plante entière, pourrait être préjudiciable à sa viabilité dans la zone. En effet, certains travaux (Thiombiano et al., 2003) l'annonçaient comme disparue de Gonsé en raison de sa surexploitation par les populations.

Dans l'optique d'optimiser la protection des denrées stockées, les producteurs utilisent de façon sélective certaines espèces comme répulsifs et d'autres comme matériaux de construction des greniers. Ainsi, les Turka font plus appel à Cassia nigricans pour conserver les céréales (maïs, mil, sorgho, riz) dans les greniers traditionnels. Selon les populations enquêtées, l'effet répulsif dû à l'odeur dégagée par cette espèce expliquerait son usage. En outre, son essence peut persister pendant plus d'un an dans le grenier. Cassia nigricans renfermerait donc des molécules actives à effets anti-appétants. Pour Maloueki et al. (2015), la présence de tanins, de saponosides, d'anthraquinones, de triterpenoïdes et de stérols serait à la base de l'activité biologique chez les plantes. La poudre des feuilles ou les écorces du tronc de Azadirachta indica et, celle des fruits de Bobgunnia madagascariensis seraient efficace dans la conservation du niébé, du maïs et du fonio. Ces résultats sont en accord avec ceux de Anjarwalla et al. (2016) qui ont montré que les gousses de Bobgunnia madagascariensis et les feuilles de Azadirachta indica sont efficaces dans la conservation des denrées alimentaires contre les termites, les charançons et les rongeurs. Quant à la poudre du broyat de feuilles et de tiges charnues de Cissus quadrangularis, elle est utilisée principalement après la récolte contre les coléoptères de postrécolte (Anjarwalla et al., 2016). En outre, nos résultats ont révélé l'usage de la cendre de certaines espèces dans la stratégie de conservation traditionnelle de céréales dans le grenier. Plusieurs travaux ont montré l'emploi de la cendre de bois dans la protection des denrées stockées. Quant à l'usage de la cendre du tronc de Khaya senegalensis, il corrobore les résultats de Toumnou et al. (2012) pour qui, cette plante est un répulsif gustatif vis-à-vis de Sitophilus oryzae. La lutte contre les bioagresseurs des plantes cultivées a été enregistrée dans plusieurs pays comme le Burkina Faso (Savadogo et al., 2016), le Sénégal (Toumnou et al.,2012) et le Togo (Bokobana et al., 2014). Pour augmenter la rigidité des greniers, à l'argile est associée de la paille de Digitaria exilis ou celle de Pennisetum glaucum. De tels constats ont été faits par (Gormo et Nizesete, 2013) lors de leurs travaux sur les structures paysannes de stockage du Cameroun. En outre, l'emploi des fruits frais pilés de Bobgunnia madagascariensis et de Neocarya macrophylla contribuerait selon les enquêtés, à inclure une odeur persistante dans le banco. Au-delà des organes végétaux, les populations utilisent l'eau de barattage du beurre de karité pour pétrir le banco. L'usage des fruits de Vitellaria paradoxa dans la lutte contre les insectes des denrées stockées a fait l'objet d'étude (Savadogo et al., 2016). Quant à Imperata cylindrica et Andropogon gayanus, elles interviennent dans la confection de la toiture du grenier. Ces espèces ont été en effet citées comme plantes de toiture des greniers traditionnels au Nord-Cameroun (Gormo et Nizesete, 2013). En outre, la plupart des espèces utilisées par les populations présentent des bonnes caractéristiques mécaniques aux attaques des insectes xylophages et aux termites. Ces critères de choix sont rapportés dans les travaux de Ganaba et al. (2004), qui ont montré que les nomades burkinabè choisissent les essences végétales pour construire les greniers traditionnels suivant les critères de solidité, de résistance aux termites et aux insectes foreurs, de facilité de travail et de port.

Cette variabilité et sélectivité des plantes, témoignent d'une bonne connaissance des plantes par les autochtones. Pour Korbéogo (2011), c'est une relation étroite entre les 
besoins utilitaires et la connaissance qu'ont les populations locales de leur environnement.

\section{Conclusion}

La présente étude chez les Turka, nous a permis de recenser 33 espèces de plantes utilisées traditionnellement pour lutter contre les bio-agresseurs des cultures et des denrées stockées. Seules ou en association, transformées ou entières, ces espèces sont efficaces dans la lutte ciblée des bioagresseurs, soit pour leur effet répulsif des insectes, soit inhibiteur des graines de mauvaises herbes ou soit dans la construction des greniers traditionnels. Ces résultats témoignent d'une bonne connaissance des plantes dans la lutte contre les bio-agresseurs et l'importance qu'occupent les ressources végétales dans les systèmes agricoles en pays Turka. Une bonne application permettrait de réduire l'impact des produits chimiques sur la santé, humaine, animale et l'environnement. En effet, plusieurs des espèces recensées auraient des impacts intéressants. Une étude approfondie des propriétés chimiques de certaines espèces pourrait réduire l'emploi des pesticides et herbicides de synthèse dans la lutte contre les bio-agresseurs.

\section{CONFLIT D'INTÉRÊTS}

Les auteurs déclarent qu'ils n'ont pas de conflit d'intérêts.

\section{CONTRIBUTIONS DES AUTEURS}

SS : rédaction $\mathrm{du}$ protocole de recherche, collecte et analyse des données et rédaction du manuscrit; $\mathrm{PZ}$ : validation du protocole de recherche, suivi de la collecte des données, validation des données analysées, instructions pour la rédaction et la correction du manuscrit; DY: validation des données analysées et lecture du manuscrit; JIB : responsable scientifique de la recherche.

\section{REMERCIEMENTS}

Nous remercions les populations et les autorités coutumières des villages enquêtés pour leurs franches collaborations.

\section{REFERENCES}

Alexiades MN. 1996. Selected guidelines for Ethnobotanical Research: A Field Manual. The New York Botanical Garden.

Andrade-Cetto A, Heinrich M. 2011. From the field into the lab: useful approaches to selecting species based on local knowledge. Frontiers in Pharmacology, 2(20): $\quad 1-4$. DOI: http://dx.doi.org/10.3389/fphar.2011.000 20

Anjarwalla P, Belmain S, Sola P, Jamnadass R, Stevenson PC. 2016. Guide des plantes pesticides. World Agroforestry Center (ICRAF), Nairobi, Kenya.

Arbonnier M. 2009. Arbres, arbustes et lianes des zones sèches d'Afrique de l'Ouest ( $3^{\text {e }}$ édition). CIRAD : Muséum national d'Histoire naturelle. Paris, 576p.

Belem M, Yameogo J, Guinko S. 2010. Les ligneux alimentaires des galeries forestières de la réserve de biosphère de la mare aux hippopotames, Burkina Faso. Fruits, Vegetable and Cereal Science and Biotechnology, 4 (1): 10-47.

Benmeddour T, Fenni M. 2018. Phytotoxicité des extraits de trois espèces végétales sur le blé dur et sur kochia scoparia: adventice envahissante des périmètres agricoles dans la wilaya de biskra. Courrier du Savoir, 25 : 173-178.

Berhaut J. 1979. Flore du Sénégal (2e édn). Clairafrique : Dakar.

Bokobana EM, Koba K, Poutouli WP, Akantetou PK, Nadio NA, Laba B, Tozoou P, Raynauld C, Sanda K. 2014. Evaluation du potentiel insecticide et répulsif de l'huile essentielle de Cymbopogon schoenanthus (L.) Spreng. sur Aphhis gossypii Glover (Homoptera : Aphididae), ravageur du cotonnier au Togo. Revue CAMES, Sciences de la vie, de la terre et agronomie, 2 (2) : 48-55.

Chérif R, Kemassi A, Boual Z, Bouziane N, Benbrahim F, Hadjseyd A, Gharib T, Ould el Hadj-Khelil A, Sakeur ML, Ould el Haj MD. 2016. Activités biologiques des extraits aqueux de Pergularia tomentosa L. (Asclepiadaceae). Lebanese 
Science Journal 17 (1) : 25-35. DOI : http://dx.doi.org/10.22453/LSJ017.1.025035

Cotton CM. 1996. Ethnobotany: Principles and Applications. Chichester: New York.

Dipama JM. 2014. La problématique de la production agricole dans un context de changement climatique. Cahiers $d u$ CERLESHS, 47: 107-125.

FAO. 2018. International Water Management Institute. More people, more food, worse water? A global review of water pollution from agriculture, FAO. 224p.

Ganaba S, Ouadba JM, Bognounou O. 2004. Plantes de construction d'habitations en région sahélienne. Bois et Forêt des Tropiques, 282(4) : 11-17. DOI : http://doi.org/1019182/bft2004.282.a202 15

Golay C. 2010. Crise et sécurité alimentaires : vers un nouvel ordre alimentaire mondial? Revue internationale de politique de Développement, 1 : 229-248. DOI : https://doi.org/10.4000/poldev.133

Gormo J, Nizesete BD. 2013. Des végétaux et leurs usages chez les peuples du NordCameroun : sélection et mode d'emploi du XIX au XX ${ }^{\mathrm{e}}$ siècle. Historia, Ciencias, Saude-Manguinho, 20(2): 587-607. DOI : https://doi.org/10.1590/S010459702013000200013.

Herrera R, Ilboudo L. 2012. Les défis de l'agriculture paysanne : le cas du Burkina Faso. L'Homme et la Société, 1-2 (183184) : 83-95. DOI : https://doi.org/10.3917//hs.183.0083

Kambou G, Somé N, Ouédraogo S. 2000. Effets des cosses de néré, Parkia biglobosa (Jacq.) R.Br. ex G.Don sur l'émergence du Striga hermonthica (Del.) Benth., les propriétés agrochimiques du sol et le rendement du maïs. Bulletin de la Recherche Agronomique, 29 : 16-30.

Korbéogo G. 2011. Les stratégies socioculturelles de conservation de la biodiversité. In Atlas de la biodiversité de l'Afrique de l'Ouest, 2 : 430-433.

INSD. 2017. Annuaire statistique 2016. Institut National de la Statistique et de la Démographie (INSD), Ministère de l'économie, des finances et du développement. 370p.

Maloueki U, Kapetshi J, Kabena NO, Ndimbo KSP, Ngbolua KNJP, Nbomba NB, Muganza CDM. 2015. Activités antimicrobiennes et antioxydantes des extraits aqueux totaux des fruits de Dialium corbisieri et Dialium gossweilerii

(FabaceaeCaesalpinioideae), consommés par les bonobos, Bolobo, République Démocratique du Congo. International Journal of Biological and Chemical Sciences, 9(2): 633-642. DOI: http://dx.doi.org/10.4314/ijbcs.v9i2.5

Olivier M, Zerbo P, Boussim IJ, Guinko S. 2012. Les plantes des galeries forestières à usage traditionnel par les tradipraticiens de santé et les chasseurs Dozo Sénoufo du Burkina Faso. International Journal of Biological and Chemical Sciences, 6 (5) : 2170-2191. DOI: 10.4314/ijbcs.v6i5.24

Rives F. 2012. Gestion des forêts sèches à Madagascar et au Niger. Vulnérabilité et fonctions des systèmes socio-écologiques pour comprendre les réformes forestières et leurs effets. Thèse de doctorat. AgroParisTech, Paris France, 303p.

Sahu A, Devkota A. 2013. Allelopathic effects of aqueous extract of leaves of Mikania micrantha H.B.K. on seed germination and seedling growth of Oryza sativa $\mathrm{L}$. and Raphanus sativus L. Scientic World, 11(11): 90-93. $\quad$ DOI: https://doi.org/10.3126/sw.v11i11.8559

Savadogo $\mathrm{S}$, Sambaré $\mathrm{O}$, Serème $\mathrm{A}$, Thiombiano A. 2016. Méthodes traditionnelles de lutte contre les insectes et les tiques chez les Mossé au Burkina Faso. Journal of Applied Biosciences, 105: 10120-10133. DOI: 10.4314/jab.v105i1.9

Teklehaymanot T. 2009. Ethnobotanical study of knowledge and medicinal plants use by the people in Dek Island in Ethiopia. Journal of Ethnopharmacology, 124 (1): 69-78.

DOI: http://doi.org/10.1016/jep.2009.04.005

Thiombiano A, Wittig R, Guinko S. 2003. Conditions de la multiplication sexuée 
chez les Combretaceae du Burkina Faso. Revue d'Ecologie (Terre vie), 58 (4) : 361-379.

Thiombiano A, Kammpman D. 2010. Atlas de la Biodiversité de l'Afrique de l'Ouest, Tome II: Burkina Faso. Ouagadougou \& Frankfurt/Main.

Thiombiano A, Schmidt M, Dressler S, Ouédraogo A, Hahn K, Zizka G. 2012. Catalogue des plantes vasculaires du Burkina Faso. Editions des conservatoires et jardins botaniques. Boissiera, 65 : 1-391.

Tiétiambou FRS, Lykke AM, Korbéogo G, Thiombiano A, Ouédraogo A. 2016. Perceptions et savoirs locaux sur les espècesoléagineuses locales dans le Kénédougou, Burkina Faso. Bois et Forêt des Tropiques, 327(1) : 39-50. DOI:

https://doi.org/10.19182/bft2016.327.a31 295

Toumnou AL, Seck DNamkosserena S, Cisse N, Kandioura N, Sembene M. 2012. Utilisation des plantes indigènes à effet insecticide pour la protection des denrées stockées contre des insectes ravageurs à Boukoko (Centrafrique). International Journal of Biological and Chemical Sciences, 6 (3): 1040-1050. DOI: 10.4314/ijbcs.v6i3.11.

Traoré L, Ouédraogo I, Ouédraogo A, Thombiano A. 2011. Perceptions, usages et vulnérabilité des ressources végétales ligneuses dans le Sud- Ouest du Burkina
Faso. International Journal of Biological and Chemical Sciences, 5 (1): 258-278. DOI: $10.4314 /$ ijbcs.v5i1.68103

Traoré O, Sérémé A, Dabiré CM, Somé K, Nébié RHC. 2015. Effet des extraits du thé de Gambie (Lippia multiflora Moldenk) et du neem (Azadirachta indica A.Juss.) sur Helicoverpa armigera et les Thrips de la tomate (Lycopersicon esculentum Mill.). Journal of applied Biosciences, 95: 89158929. DOI: $10.4314 /$ jab.v95i1.2

Yarou BB, Silvie P, Komlan FA, Mensah A, Alabi T, Verheggen F, Francis F. 2017. Plantes pesticides et protection des cultures maraichères en Afrique de l'Ouest (synthèse bibliographique). Biotechnologie, Agronomie, Société et Environnement, 21 (4) : 288-304. DOI : https://doi.org/10.25518/17804507.16175

Zerbo P, Millogo-Rasolodimby J, Nacoulma OG, Van Damme P. 2011. Plantes médicinales et pratiques médicales au Burkina Faso: cas de Sanan. Bois et Forêts des Tropiques 307 (1) : 37-53. DOI: https://doi.org/10.19182/bft2011.307.a20 481

Zerbo P, Compaoré M, Méda NR, LamienMéda A, Kiendrebéogo M. 2013. Potential Medicinal plants used by traditional healers in western areas of Burkina Faso. World Journal Pharmacy and Pharmaceutical Sciences, 2(6): 6706-67619. 